\title{
A COMPARISON OF IMAGE SEGMENTATION TECHNIQUES, OTSU AND WATERSHED FOR X-RAY IMAGES
}

\author{
P. Manju Bhargavi ${ }^{1}$, V. Sai Kiran Mayee ${ }^{2}$, T. Manaswini ${ }^{3}$, S. Manvitha ${ }^{4}$ \\ ${ }^{1}$ Department of Biomedical Engineering, GRIET, Hyderabad \\ ${ }^{2}$ Department of Biomedical Engineering, GRIET, Hyderabad \\ ${ }^{3}$ Department of Biomedical Engineering, GRIET, Hyderabad \\ ${ }^{4}$ Department of Biomedical Engineering, GRIET, Hyderabad
}

\begin{abstract}
The most dangerous and rapidly spreading disease in the world is Tuberculosis. In the investigating for suspected tuberculosis (TB), chest radiography is the only key techniques of diagnosis based on the medical imaging So, Computer aided diagnosis $(C A D)$ has been popular and many researchers are interested in this research areas and different approaches have been proposed for the TB detection. Image segmentation plays a great importance in most medical imaging, by extracting the anatomical structures from images. There exist many image segmentation techniques in the literature, each of them having their own advantages and disadvantages. The aim of X-ray segmentation is to subdivide the image in different portions, so that it can help during the study the structure of the bone, for the detection of disorder. The goal of this paper is to review the most important image segmentation methods starting from a data base composed by real X-ray images.
\end{abstract}

Keywords - chest radiography, computer aided diagnosis, image segmentation, anatomical structures, real X-rays. $* * *$

\section{INTRODUCTION}

Tuberculosis (TB) is an infectious disease over the world population. It is caused by bacteria which is known as Mycobacterium tuberculosis and mostly affected to the lungs of the human beings. In order to detect Tuberculosis, we have set of steps involved [1].

A CAD system usually applies a series of pre-processing steps to an input image. The pre-processing step is performed to enhance the image quality so that objects of interest become more evident. The quality of the preprocessing step strongly affects the performance of the subsequent processing steps. The pre-processing steps for X-ray screening are contrast enhancement and lung boundary detection. The software implementation of these steps is shown in this paper.

When analyzing objects in images, it is necessary to distinguish the objects of interest from the background. This task can be realized through segmentation. Image segmentation is one of the most challenging issues in image processing domain [2] and it has been an active research area in the last years. Through segmentation we aim to fragment the image in a series of regions, based on the attributes of the image that are approximately constant in each region, but differ significantly from a region to another. Segmentation aims to extract useful information from images in medical imaging applications as well Medical images segmentation [2] has also found applications in studying the anatomical structure, fractures localizations or tumors, diagnosis and treatment planning, computerintegrated surgery, tissue classification, or tumor volume estimation. They are also affected by noise, artifacts or spatial aliasing so that the boundaries of the regions of interest to become indistinct. X-rays are having various orientations, resolutions, and luminous intensities, depending on the X-ray equipment, which influences the quality of the segmentation result [3]. Unlike many other medical imaging modalities, bone regions often overlap with other organs or bones and the joints between bones in Xrays. Several segmentation techniques have been developed and reported in the literature. However, a perfect method, which is universally applicable to all kind of images, does not exist. This paper focuses on the comparison of several already existing image segmentation techniques in the case of X-ray images.

\section{WORK}

\subsection{Pre-Processing:}

The objective of the image pre-processing is not only to improve the image quality but also to reduce the undesired portion from the background of the images. Most preprocessing methods apply the intensity value of neighborhood pixel for obtaining the brightness intensity value of the input images. The reasons for the image preprocessing phase are:

i. In order to meet the requirements of the physician for the image quality improvement.

ii. Edge detection -To detect the edges, Sobel operator is discussed in this paper.

iii. Contrast enhancement - To enhance the contrast at the region of interest, histogram equalization is performed on the image. Histogram analysis increases the contrast in low-contrast regions by spreading out the most frequent intensity levels. 
iv. Preparing the data optimally for post-processing steps. The process is described in figure 1.

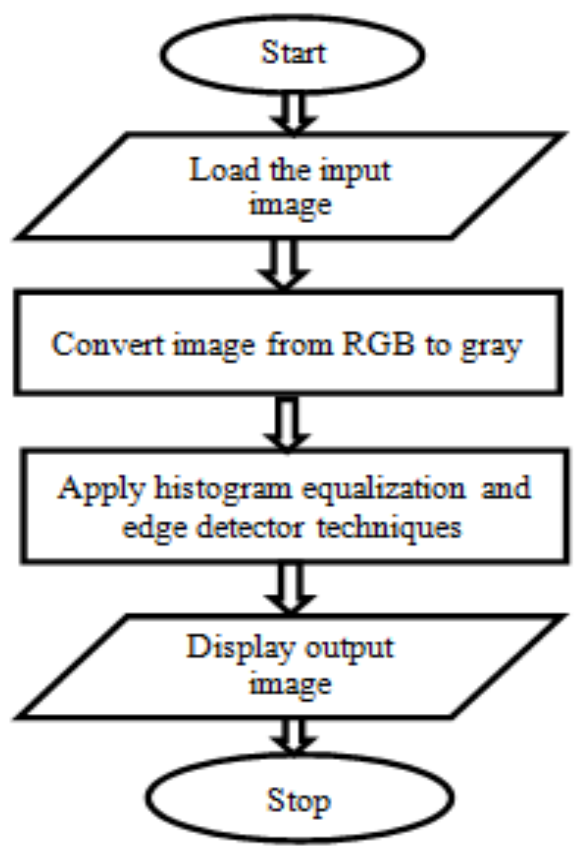

Fig 1: Flowchart of pro-processing step

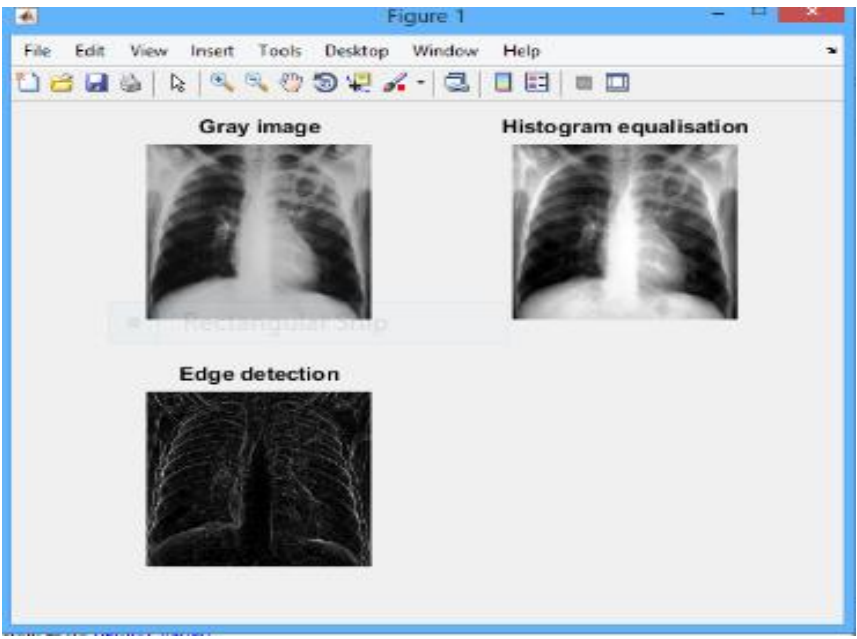

Fig 2: Output of pre-processing step

In figure 2, the first image is the input which is a gray image converted from RGB image. The second is histogram equalized image and the last is the edge detected image using a Sobel operator.

\subsection{Segmentation Techniques}

Image segmentation is very important step to extract good quality features for classification. The objective of image segmentation is to cluster the pixels having same intensity value from the whole image regions, separating regions of desired part of the original image, hiding the undesired region. Image segmentation step is applied in many image pre-processing areas such as editing, or query from image database, object occlusion, recognition of object, estimation of the boundary and image compression.
In general, algorithms for image segmentation are based on two fundamental properties of intensity values which are discontinuity and similarity. Discontinuity property does the partition of the image based on the changes in sharp intensity value. Similarity property does the partition of the image into regions that are alike to the specified criteria.

In chest radiographs, segmentation process must be done accurately for the medical image analysis task which is especially for computer-aided diagnosis. For example, lung nodules are determined according to the different measurements of nodules size.

Different kinds of approaches have been discussed in the lung segmentation step. Various approaches for lung segmentation are presented which includes:

\subsubsection{Watershed Segmentation}

Watershed segmentation (watershed transform) is a regionbased method [4] under the classical technique of segmentation. This method is based on the grayscale mathematical morphology [5] and it is used for multi component images. Intuitively, the watershed algorithm can be thought of as a landscape that is flooded by water. At each point, the height of the landscape represents the pixel's intensity. The watershed transform computes the image regions which represent the basins and region boundaries (the ridgelines). The image gradient is used as input of the transform, such that the basin limits are situated at high gradient points. The steps involved in this method are shown in figure 3.

A Watershed segmentation process starts at some regional minima $\mathrm{M}_{\mathrm{i}}$ value which locates the lowest points of the area into where the water flows. By measuring appropriate distance, the area is separated into regions $\Omega \mathrm{i}$ which has growth from the corresponding minimum $\mathrm{M}_{\mathrm{i}}$ by adding to $\Omega_{\mathrm{i}}$, repeatedly, unlabeled points on the outer boundary of $\Omega_{\mathrm{i}}$. A point is added to region $\Omega_{\mathrm{i}}$ If its distance from the region is smaller than those from other regions. The process is repeated until no remaining unlabeled points are those of the watershed line. In implementation, in order to obtain a thinwatershed line, a point is added to the region $\Omega i$ even when its distance from the region equals those from some other regions. Hence, there is no point that belongs to the watershed line.

This type of segmentation method is very simple and intuitive and has good properties, which make it useful for many image segmentation applications. However, it has other disadvantages such as: over-segmentation and poor at detecting thin structures and structures with low signal-tonoise ratio. 


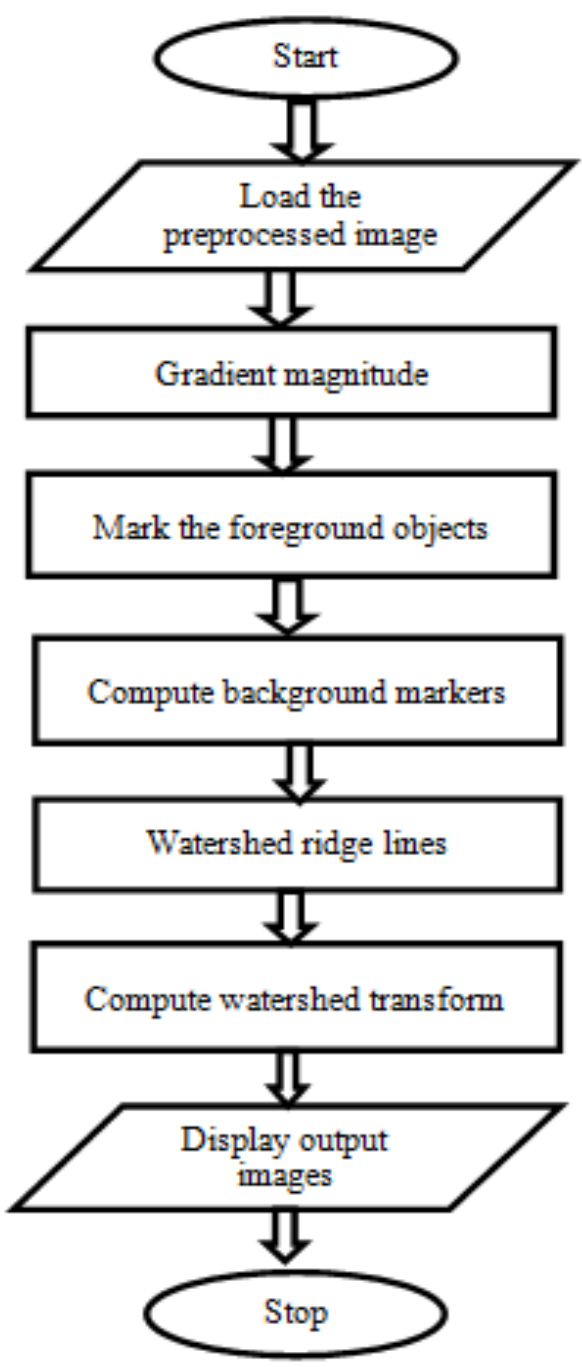

Fig 3: Flowchart of Watershed segmentation

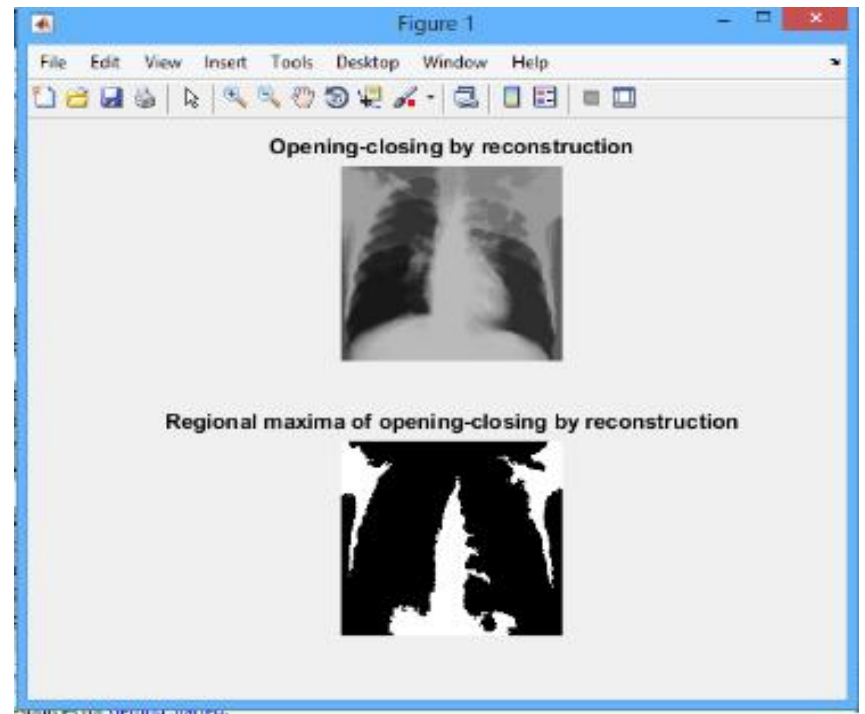

Fig 4: Output of reconstruction technique

In figure 4, the first image is opening and closing technique by reconstruction and the next image is the regional maxima of opening and closing technique by reconstruction.

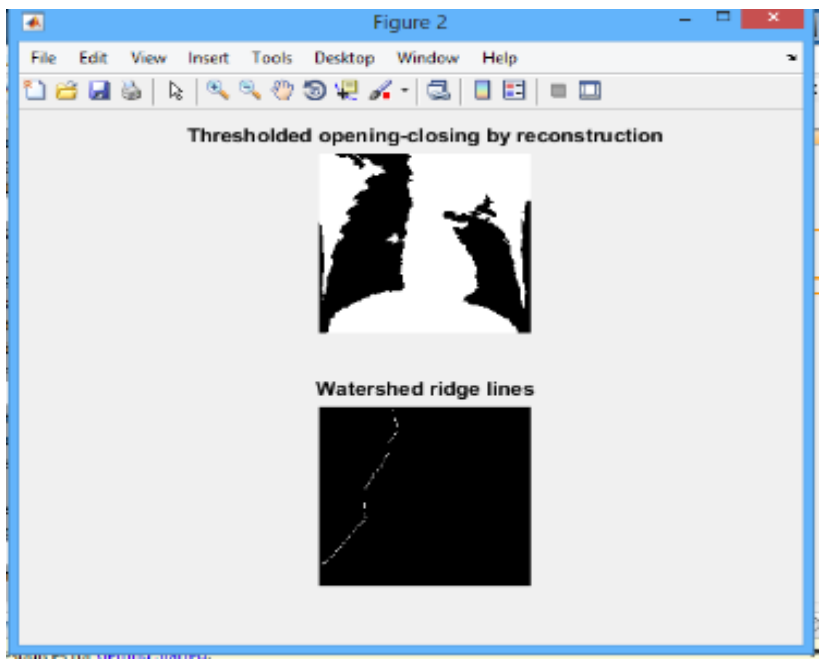

Fig 5: Output of thresholding technique

In figure 5, the first image is by applying a threshold to the opening-closing reconstruction technique and the next image is the watershed ridge line.

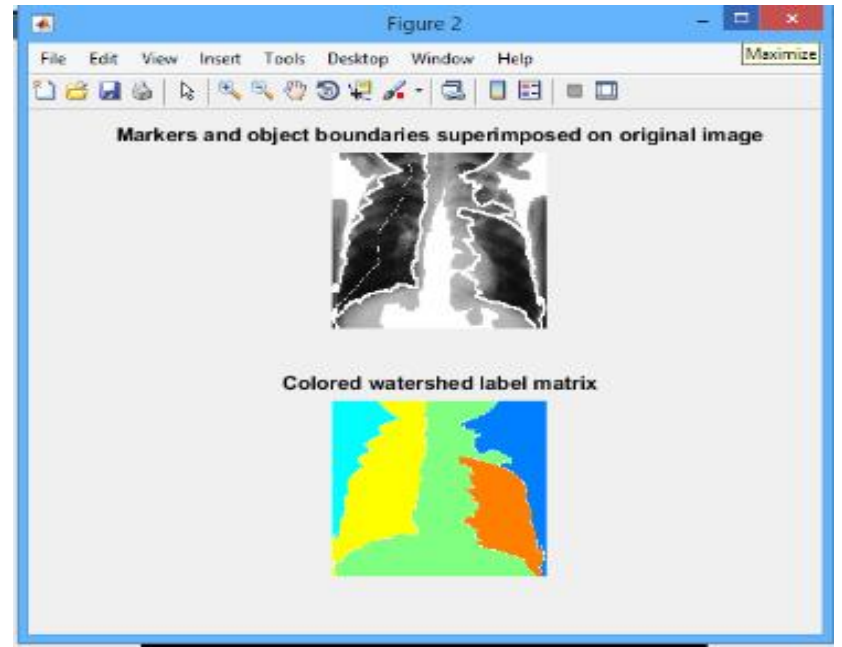

$\overline{\text { Fig 6: Output of watershed segmentation }}$

In figure 6, markers and object boundaries are superimposed on an original image and the last image is the coloured watershed label matrix.

\subsubsection{Otsu Thresholding}

Thresholding is one of the most simple segmentation techniques [2], involves thresholding the intensity of the image. In the case of global thresholding, only one threshold value is selected for the entire image. Global methods are based on the fact that the image has a bimodal histogram. The region of interest can be separated from the background by comparing the intensity of each pixel in the image with a threshold. Some pixels, whose intensity values are greater than the threshold, are classified as being part of group A object of interest (with an intensity value of 1 ), and the rest of the pixels as being part of group B-background (with an intensity value of 0 ). The following assumptions are applied in Otsu method: 


\begin{abstract}
i. The image has a bimodal histogram.
ii. Neither use of spatial coherence nor any other object structure.

iii. Intensity values are stationary statistics but they can be changed to locally adaptive.

iv. Intensities are implicitly uniform illumination thus the brightness behavior of the bimodal arises from the object appearance differences only.
\end{abstract}

Global methods are simple and fast, but are suitable only for images with bimodal intensity distribution (probability distribution with two different modes). Another factor that affects the performance of thresholding is the unequal illumination in the image. In addition, global methods are not useful for multichannel images, since only two classes are generated. Figure 7 shows the procedure involved in otsu thresholding.

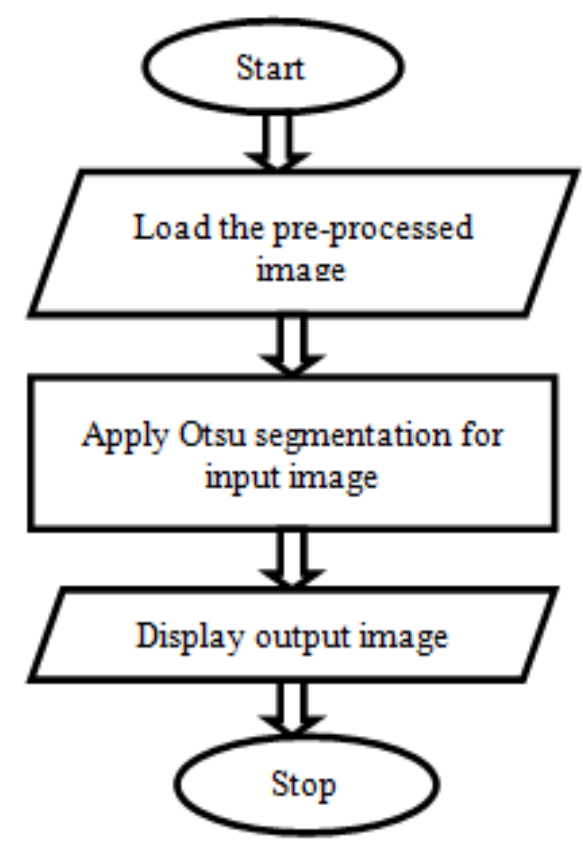

Fig 7: Flowchart of Otsu thresholding technique

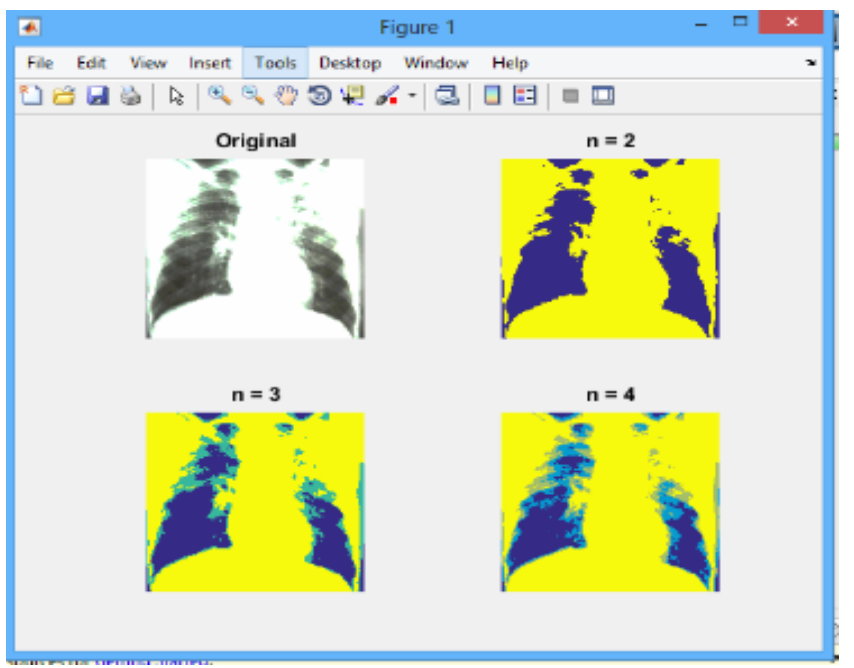

Fig 8: Output of Otsu thresholding technique
In figure 8 , the first image is the original image that is given as the input which is actually pre-processed and the next three images are obtained by setting different threshold values to the input image.

\section{CONCLUSION}

We described a series of approaches that have been published in the recent literature, concerning medical images segmentation. We have provided an overview regarding the implementation of each segmentation method, highlighting the advantages and disadvantages of each method. The evaluation of these segmentation techniques can be done, in terms of: performance, computational complexity and sensitivity to noise. The most accurate techniques are the most complex and time consuming. We realized the classification starting with the most simple and fast methods and we increased the computational complexity and the processing time with each presented method.

As future work, we intend to combine a classical image segmentation technique for an initial segmentation and then to apply a deformable model in order to increase the segmentation accuracy. We intend to calculate the region of interest (ROI) on the segmented regions and then apply a classifier technique in order to detect the Tuberculosis and if present, we intend to find the intensity of its spread to different regions in the thoracic cavity.

\section{REFERENCES:}

[1] S. V.Zharkova, EGSO, 29 october 2002. [Online]. Available:

https://www.google.co.in/url?sa=t\&rct=j\&q=\&esrc=s $\&$ source $=$ web $\& \mathrm{~cd}=2 \& \mathrm{cad}=\mathrm{rja} \&$ uact $=8 \&$ ved $=0 \mathrm{CCQ}$ QFjAB\&url=http\%3A\%2F\%2Fwww.researchgate.ne t\%2Fprofile\%2FValentina_Zharkova\%2Fpublication \%2F250738597_Survey_of_Image_Processing_Tech niques\%2Flinks\%2F5488d3ae0cf268d28. [Accessed february 2015].

[2] G.Dougherty, "Medical Image Processing Techniques and Applicatios," Springer2011.

[3] D. W. k. D.Feng, july 2006. [Online]. Available: http://www.comp.nus.edu.sg/ leowwk/thesis/dingfen g-proposal.pdf. [Accessed march 2015].

[4] S. V. Kasmir Raja, A. Shaik Abdul Khadir, and S. S. Riaz Ahamed, "Moving toward region-based image segmentation techniques: a study", Journal of Theoretical and Applied Information Technology, 5:81-87, 2009.

[5] S. S.K.Mahendran, "Enhanced automatic X-ray bone image segmentation using wavelets and morphological operators," 2011.

[6] M. W.Burgern, "Principles of Digital Image Processing Fundamental Techniques," Springer, 2009. 